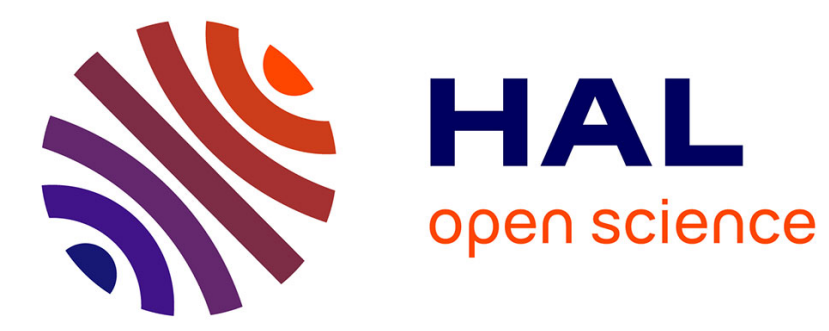

\title{
An ellipsometry study of silica nanoparticle layers at the water surface
}

Duyang Zang, Antonio Stocco, Dominique Langevin, Bingbo Wei, Bernard P Binks

\section{- To cite this version:}

Duyang Zang, Antonio Stocco, Dominique Langevin, Bingbo Wei, Bernard P Binks. An ellipsometry study of silica nanoparticle layers at the water surface. Physical Chemistry Chemical Physics, 2009, 11 (41), pp.9522-9. hal-00785456

\section{HAL Id: hal-00785456 https://hal.science/hal-00785456}

Submitted on 6 Feb 2013

HAL is a multi-disciplinary open access archive for the deposit and dissemination of scientific research documents, whether they are published or not. The documents may come from teaching and research institutions in France or abroad, or from public or private research centers.
L'archive ouverte pluridisciplinaire HAL, est destinée au dépôt et à la diffusion de documents scientifiques de niveau recherche, publiés ou non, émanant des établissements d'enseignement et de recherche français ou étrangers, des laboratoires publics ou privés. 


\title{
An ellipsometry study of silica nanoparticle layers at the water surface
}

\author{
Duyang Zang, ${ }^{1,2}$ Antonio Stocco, ${ }^{*}{ }^{1}$ Dominique Langevin, ${ }^{1}$ Bingbo $\mathrm{Wei}^{2}$ \\ and Bernard P. Binks ${ }^{3}$
}
1. Laboratoire de Physique des Solides, Université Paris-Sud, UMR CNRS 8502, Bâtiment 510, 91405 Orsay cedex, France.

2. Laboratory of Materials Science in Space, Northwestern Polytechnical University, Xi'an, 710072, China.

3. Surfactant \& Colloid Group, Department of Chemistry, University of Hull, Hull, HU6 7RX, UK.

\begin{abstract}
We have studied silica nanoparticle layers spread at the air-water interface. The surface pressure of the layers has been determined in a Langmuir trough via two orthogonal Wilhelmy plates. We observed significant differences in surface pressure according to the preparation protocol: layers spread then compressed or layers obtained after successive spreading steps. We also studied the two types of layers by multiple angle of incidence ellipsometry. We introduce a two-layer model which enables us to evaluate the radius of interfacial aggregates and their contact angle with the air-water interface.
\end{abstract}

Keywords: nanoparticle, spread layer, air-water interface, ellipsometry, contact angle. 


\section{Introduction}

Nanoparticles can adsorb at the air-water and oil-water interfaces when they are partly hydrophobic forming surface layers which are nowadays the object of much interest. ${ }^{1-8}$ The energy of adsorption $W$ is related to the contact angle $\theta$ of the particle with the interface: $W=$ $\gamma_{0} R^{2} \pi(1 \pm \cos \theta)^{2}$ for spherical particles, where $R$ is the radius of the particle and $\gamma_{0}$ the surface tension of the bare interface (free of particles). When $\theta \sim 90^{\circ}, W \sim(R / a)^{2} k T, a$ being a molecular size, $k$ the Boltzmann constant and $T$ the absolute temperature, indicating that since $W>>T$, the adsorption is irreversible. ${ }^{5}$ Particles can organise differently at interfaces: a wellordered 2-D structure was first observed for 122.5 nm-radius polystyrene latex particles, ${ }^{5}$ bonded particle clusters were seen for charged micron size polystyrene spheres, ${ }^{9}$ and disordered structures were observed for silica microparticles at the oil-water interface. ${ }^{10}$ These different structures reflect the different interactions ${ }^{6,11,12}$ between particles at the interface. Besides attractive capillary interactions, spherical charged colloidal particles can experience either Coulombic repulsion because of their vertically asymmetric charge distribution or long range attraction due to non-uniform horizontal charge distribution. ${ }^{9}$ Moreover, effective interactions due to non-linear charge renormalization can play an important role in the case of non-spherical particles. ${ }^{11}$ Many other phenomena such as 2-D aggregation, ${ }^{13-15}$ order-disorder transitions $s^{7,10,16}$ and wetting behaviour ${ }^{17}$ are under investigation for colloidal particles at fluid interfaces.

Besides the intrinsic fundamental interest, nanoparticles allow one to produce extremely stable foams or emulsions, with possible interesting applications. ${ }^{18}$ In the case of foams for instance, unlike most surfactants and polymers, oxide particles can be used at high temperature to stabilise metallic foams. ${ }^{19}$ Emulsions stabilised by particles (Pickering emulsions) have been extensively investigated for the production of nanomaterials. ${ }^{20}$ Partially 
hydrophobic nanoparticles are interesting model systems for studying the stability of emulsions and foams. ${ }^{21-27}$ They can be either spread or adsorbed at the interfaces from aqueous dispersions. The properties of both types of layers have been investigated in the case of silica nanoparticles and it was shown that stable foams could be produced when $E>\gamma / 2, E$ being the surface compression elastic modulus and $\gamma$ the surface tension (Gibbs stability criterion). ${ }^{23,28}$

A first ellipsometry study of spread layers of fumed silica nanoparticles at the airwater interface was performed by Safouane et al. with a conventional (single-angle measurement) ellipsometer. ${ }^{22}$ A surprising independence of the thickness and refractive index of the layer on surface coverage by particles was reported. In order to clarify the layer structure, we undertook a new study described here. We studied two types of spread layers: layers spread then compressed, called hereafter "compressed layers" or layers obtained after successive spreading steps, called hereafter "deposited layers". To measure the surface pressure $\Pi=\gamma_{0}-\gamma$, we used two different troughs: a rectangular Langmuir trough equipped with two orthogonal Wilhelmy plates and a circular trough. We also used multiple angle of incidence ellipsometry to measure the layer thickness and the particle surface coverage, which enabled us to evaluate the contact angle $\theta$.

\section{Materials, preparation and methods}

\subsection{Materials}

The silica particles were kindly provided by Wacker-Chemie (Germany) and were used as received. The surfaces of the particles were modified by reaction of the surface silanol groups with dichlorodimethylsilane. In this study, we used particles with a relative $\mathrm{SiOH}$ content of $20,34,51$ and $62 \%$, in which the hydrophobicity increases with decreasing $\mathrm{SiOH}$ content. 
The latter content was determined by a titration technique using strong base. We focused in particular on the $34 \% \mathrm{SiOH}$ system, because at this intermediate hydrophobicity the most stable aqueous foams were observed. ${ }^{25}$ Mainly, we compared the $34 \% \mathrm{SiOH}$ particle system with both a highly hydrophobic $(20 \% \mathrm{SiOH})$ and a more hydrophilic $(62 \% \mathrm{SiOH})$ system. The particles are clusters of irreversibly bound primary silica particles, which are spherical-like objects of approximately $20 \mathrm{~nm}$ diameter. The dispersions were prepared by adding amorphous fumed silica powder into isopropyl alcohol to a final concentration of $1 \mathrm{~g} \mathrm{~L}^{-1}$. The dispersion was sonicated for $30 \mathrm{~min}$. using an ultrasonic probe (Ultrasonic Processor) operating at $20 \mathrm{kHz}$ with an amplitude of $45 \%$ of the maximum in order to have a stable dispersion and to avoid the formation of particle aggregates. The dispersion was re-sonicated for $10 \mathrm{~min}$. just before each experiment.

Dynamic light scattering experiments were performed in order to characterize the size and polydispersity of the particle aggregates (in water ${ }^{23}$ and) in isopropyl alcohol using the method of cumulants. ${ }^{29}$ Autocorrelation functions were fitted by $f^{m}(q, \tau)=\exp (-$ $\left.<D>q^{2} \tau\right)\left(1+1 / 2 p\left(-<D>q^{2} \tau\right)^{2}\right)$, where $D$ is the diffusion coefficient, $q$ is the scattering vector, $\tau$ is the correlation time and $p$ is a polydispersity index. The hydrodynamic radius $R_{h}$, calculated by the Stokes-Einstein equation, changes as a function of the $\% \mathrm{SiOH}$. The following values were found: $R_{h}=87 \pm 2 \mathrm{~nm}$ for $20 \% \mathrm{SiOH}, R_{h}=77 \pm 1 \mathrm{~nm}$ for $34 \% \mathrm{SiOH} . R_{h}=72 \pm 2 \mathrm{~nm}$ for $62 \% \mathrm{SiOH}$. These fumed silica dispersions were also fairly monodisperse in isopropyl alcohol, the second order polydispersity index $p=\left(\left\langle D^{2}\right\rangle-\langle D\rangle^{2}\right) /\langle D\rangle^{2}$ being between 0.11 and 0.21. Water used in the experiments was ultra-purified water from a Millipore-Q instrument (resistivity $=18 \mathrm{M} \Omega \mathrm{cm}$ ). All the experiments described in the paper were performed at room temperature $\left(\sim 22^{\circ} \mathrm{C}\right)$. The particle dispersion was spread onto the airwater interface drop by drop from a microlitre syringe. 


\subsection{Compressed layers}

The compressed layers were made by spreading an initial amount of particle dispersion at the surface of water contained in a rectangular Langmuir trough (Nima 601BAM, total area $500 \mathrm{~cm}^{2}$ ). The trough was cleaned with ethanol and pure water before each experiment. Before spreading the particles, the surface of pure water was compressed and it was checked that the surface pressure $\Pi$ did not exceed $0.3 \mathrm{mN} \mathrm{m}^{-1}$ during compression, ensuring the absence of contamination. After spreading a certain volume (usually $600 \mu \mathrm{L}$ ) of particle dispersion in isopropyl alcohol onto the air-water interface, we waited for $30 \mathrm{~min}$. to allow the solvent to evaporate.

The nominal surface concentration $\Gamma$ increases when reducing the area by closing the barriers. The variation of the surface pressure with $\Gamma$ was monitored by two Wilhelmy plates: one parallel to the barriers and the other perpendicular to them, the corresponding pressures being $\Pi_{\|}$and $\Pi_{\perp}$ respectively. In each experiment, the two barriers moved simultaneously. In continuous compression experiments, $\Pi-\Gamma$ isotherms were obtained by closing the barriers $(\Gamma$ was varied from 10 to $120 \mathrm{mg} \mathrm{m}^{-2}$ ) at a constant rate of $25 \mathrm{~cm}^{2} \min ^{-1}$ or $7 \mathrm{~cm}^{2} \mathrm{~min}^{-1}$. We measured the surface pressure $\Pi=\gamma_{0}-\gamma$ for continuously compressed layers in a Langmuir trough.

\subsection{Deposited layers}

We prepared these layers either in the Langmuir trough (Nima 601BAM) or in a homemade teflon circular trough (diameter $=8 \mathrm{~cm}$, depth $=3 \mathrm{~cm}$ ). The area of the liquid surface is kept constant and the nominal surface concentration $\Gamma$ is varied by successive additions of particle dispersion. Before spreading the dispersion onto the air-water interface in the circular trough, the surface tension of pure water was measured: a value of $\gamma_{0}=72.5 \mathrm{mN} \mathrm{m}^{-1}$ (within 
experimental accuracy, $\sim 0.1 \mathrm{mN} \mathrm{m}^{-1}$ ) ensured the purity of the water and the cleanliness of the trough. The particle dispersion was added with a micro-syringe drop by drop onto the water surface.

\subsection{Brewster angle microscopy}

The texture of particle layers was observed by a Brewster Angle Microscope (MiniBAM, NFT-Nanofilm Technology, Göttingen). The BAM is equipped with a high-power red laser diode (wavelength $688 \mathrm{~nm}$ ), a polarizer and an analyzer. The images taken at the Brewster angle of $53.1^{\circ}$ were recorded by means of a video recorder.

\subsection{Ellipsometry}

We performed multiple angle of incidence (MAI) measurements ${ }^{30}$ using an imaging ellipsometer (Nanofilm, Germany) working with green laser light $(\lambda=532 \mathrm{~nm})$. A fixed compensator $\left(= \pm 45^{\circ}\right)$ and 4-zone averaging nulling scheme were adopted. We used either a circular trough $($ diameter $=8 \mathrm{~cm}$, depth $=3 \mathrm{~cm})$ or a small Langmuir trough (total area 100 $\mathrm{cm}^{2}$ ) for the measurements. The surface of water was cleaned several times and ellipsometric scans were performed at the bare air-water interface before spreading the particle layer. We waited at least two hours after spreading before measuring the ellipsometric angles $\Psi$ and $\Delta$ (around the Brewster angle $\varphi_{B}=53.1^{\circ}$ for the air-water interface).$^{30}$ In order to minimize effects due to external vibrations on the measurements, the troughs were placed on top of an active vibration isolation table (Halcyonics, Germany). The data allowed us to obtain information on the interfacial profile, in particular on the thickness and refractive index of the surface layer. $^{31}$

All experiments were carried out at $22 \pm 2{ }^{\circ} \mathrm{C}$. 


\section{Results and Discussion}

\subsection{Surface pressure}

The surface pressures $\Pi_{\|}$and $\Pi_{\perp}$ were measured in the Langmuir trough. The results for the layer comprising $34 \% \mathrm{SiOH}$ particles are illustrated in Figure 1 . For all $\% \mathrm{SiOH}$, the pressure starts to increase above $20 \mathrm{mg} \mathrm{m}^{-2}$ and tends to saturate around $50 \mathrm{mg} \mathrm{m}^{-2}$. It is worth noting that the pressure measured by the parallel sensor $\left(\Pi_{\|}\right)$is always higher than that measured by the perpendicular one $\left(\Pi_{\perp}\right)$. This large difference between the two reveals the existence of a non-zero shear modulus. ${ }^{32-34}$ Here, the relaxation times of the surface layer are very long $\left(>10^{4} \mathrm{~s}\right)$, and during the continuous compression, the layer has no time to relax, hence the difference between the two pressures. ${ }^{35}$ Decreasing the barrier speed from 25 to 7 $\mathrm{cm}^{2} \min ^{-1}$ causes this difference to decrease, but it still remains non-zero. This is because relaxation can take place, but remains incomplete. ${ }^{36}$

For continuously compressed layers, the textural evolution was monitored by Brewster angle microscopy. The results are illustrated in Figure 2. At small $\Gamma\left(<20 \mathrm{mg} / \mathrm{m}^{2}\right)$, Brewster angle microscopy evidences the presence of isolated islands, with the incomplete coverage of the water surface reflecting the almost zero surface pressure, as shown in Figure 2(a). Upon increasing $\Gamma$, the layer becomes homogeneous and the surface pressure changes (see Figure 2(b)). If $\Gamma$ is further increased, small ripples nucleate in the region where the slope $\mathrm{d} \Pi / \mathrm{d} \Gamma$ is maximum (Figure 2(c)). When the pressure is further increased, the ripples evolve toward a significant buckling, as illustrated in Figure 2(d). These textural observations are as reported earlier at the air-water interface ${ }^{22}$ and oil-water interface for the same particles. ${ }^{21}$ For large $\Gamma$, the surface pressure almost saturates; however the values obtained are questionable, because they are measured in the presence of buckling. 
In Figure 3 we show the scenario described above. In this experiment, we stopped the compression at the nominal surface concentration of $60 \mathrm{mg} \mathrm{m}^{-2}$ and we monitored the change in surface pressure and layer texture: the surface pressure decreases with time while buckling tends to disappear. This observation confirms that the layer is out of equilibrium.

The surface pressures of layers obtained after successive spreading are also shown in Figure 1. They are close to $\Pi_{\perp}$ for continuously compressed layers measured by the orthogonal plate. Note that there is also a difference between $\Pi_{\|}$and $\Pi_{\perp}$ for the deposited layers although these layers were not compressed with the barriers: it is likely that compression stresses arose during spreading in the rectangular trough. The surface pressures in the circular trough are also comparable to those measured with adsorbed layers (from bulk) obtained with aqueous particle dispersions. ${ }^{23}$ BAM images of these layers were also taken. Figure 4 shows the case of $34 \% \mathrm{SiOH}$. The layers are very homogeneous, except at large concentrations where thicker regions are seen close to the trough boundaries.

The same experiments were also performed with particles with different \% $\mathrm{SiOH}(20$, and $62 \%$ ). The $\Pi-\Gamma$ curves are similar (as in ref. 22) and anisotropic effects are always observed: parallel and orthogonal pressures always differ. Furthermore, the surface pressures measured for deposited layers are always close to those for compressed layers measured by the orthogonal plate. These differences point to the important role played by the preparation protocol.

\subsubsection{Ellipsometry - Deposited layers}

In ellipsometry, the well-known equation $r_{p} / r_{s}=\tan \Psi \exp (i \Delta)$ relates the ratio of the Fresnel reflection coefficients $r$ in $p$ and $s$ polarization with the measured ellipsometric angles $\Psi$ and $\Delta$ at a given incident angle $\varphi$. Ellipsometry is sensitive to the dielectric profile across 
the interface. For air-liquid interfaces, the ellipsometric signal can result from several contributions: surface roughness driven by thermal fluctuations (capillary waves), presence of adsorbed species at the interface and optical anisotropy of interfacial material. Here the particles are spherical-like, so no optical anisotropy contribution is expected. The particle layers have a thickness well above the amplitude of capillary waves $(<1 \mathrm{~nm})$ and dominate the ellipsometry signal.

A stratified homogeneous layer model is usually adopted to describe the interfacial profile. ${ }^{30}$ Let us first discuss the case of successively deposited layers. We show in Figure 5(a) the ellipsometric scans from layers of particles of different $\mathrm{SiOH}$ content. Both the minimum $\Psi$ and the slope in $\Delta$ far from the Brewster angle increase with particle hydrophobicity (decreasing \% SiOH). Qualitatively, these changes correspond to either a surface density or a layer thickness increment with increasing particle hydrophobicity.

We first fitted the experimental data assuming a single homogeneous interfacial layer characterized by a refractive index $n_{l}$ and a thickness $d$ between the air $\left(n_{A i r}=1\right)$ and water $\left(n_{H 2 O}=1.333\right)$ macroscopic phases. ${ }^{30}$ We could extract both $n_{l}$ and $d$ since we adopted a MAI scheme. ${ }^{31}$ For particles possessing $62 \% \mathrm{SiOH}$, we obtained: $n_{l}=1.345 \pm 0.001$ and $d=$ $126 \mathrm{~nm}$ for $\Gamma=40 \mathrm{mg} \mathrm{m}^{-2}, 165 \mathrm{~nm}$ for $\Gamma=80 \mathrm{mg} \mathrm{m}^{-2}$ and $134 \mathrm{~nm}$ for $\Gamma=160 \mathrm{mg} \mathrm{m}^{-2}$. For particles with $34 \% \mathrm{SiOH}, n_{l}=1.355 \pm 0.002$ and $d=184 \pm 1 \mathrm{~nm}$; for particles with $20 \%$ $\mathrm{SiOH}, n_{l}=1.420 \pm 0.019$ and $d=205 \pm 1 \mathrm{~nm}$. The thicknesses are similar to those reported by Safouane $e t a l .{ }^{22}(d \sim 230 \mathrm{~nm})$ although $n_{l}$ was fixed to a somewhat larger value: $n_{l}=1.465$. However, the accuracy of the earlier measurements is far below that of the new ones. More importantly, the fact that the thickness and refractive index were independent of surface coverage seems to be confirmed. 
From those values, it is also possible to extract the surface concentration "seen" by ellipsometry assuming a linear change of the refractive index with concentration, i.e. ${ }^{37}$

$$
\Gamma_{\text {elli }}=\left(n_{l}-n_{H 2 O}\right) /(\partial n / \partial c) d
$$

where $(\partial n / \partial c)$ is the refractive index increment in water. It can be measured experimentally or calculated as $n_{\mathrm{SiO2}}-n_{\mathrm{H} 2 \mathrm{O}}$, where $n_{\mathrm{SiO} 2}=1.475$ is the bulk refractive index of the silica nanoparticle ${ }^{38}$ and $n_{H 2 O}=1.333$. It is worth noting that Eq. 1 assumes that the particles are totally immersed in water, being the refractive index contrast calculated as $\left(n_{l}-n_{\mathrm{H} 2 \mathrm{O}}\right) /\left(n_{\mathrm{SiO} 2}\right.$ $n_{\mathrm{H} 2 \mathrm{O}}$ ). Equation 1, assuming a linear change of the refractive index with concentration, is largely used in the literature because of the proven experimental linearity between $\operatorname{Im}\left(r_{p} / r_{s}\right)$ and $\Gamma_{.}^{31}$ Alternatively, from the Lorenz-Lorenz equation, Cuypers et $a l .^{39}$ calculated the surface concentration using:

$$
\Gamma_{\text {elli }}=\frac{\frac{n_{l}+n_{\mathrm{H} 2 O}}{\left(n_{l}^{2}+2\right)\left(n_{H 2 O}^{2}+2\right)}}{\frac{A}{M_{S i O 2}}-v \frac{n_{H 2 O}^{2}-1}{n_{H 2 O}^{2}+2}}\left(n_{l}-n_{H 2 O}\right) \times 3 d \text {. }
$$

where $A / M_{\mathrm{SiO} 2}=0.128 \mathrm{~mL} \mathrm{~g}^{-1}$ and $v=0.45 \mathrm{~mL} \mathrm{~g}^{-1}$ are the ratio of molar refractivity $(A)$ to molecular weight $\left(M_{\mathrm{SiO} 2}\right)$ and the specific volume of the adsorbed particles, respectively. We plot the calculated surface concentration $\Gamma_{\text {elli }}$ from Eqs. 1 and 2, without noting a significant difference between the two calculations, in Figure 5(b) together with the line $\Gamma_{\text {elli }}=\Gamma$. As was qualitatively observed in the ellipsometric scans of Figure 5(a), the layers of particles with $62 \% \mathrm{SiOH}$ have an apparent low surface concentration $\Gamma_{\text {elli }}<\Gamma$. On the contrary, the calculated surface concentrations for particle layers with $20 \% \mathrm{SiOH}$ are too high: $\Gamma_{\text {elli }}>\Gamma$. If all the particles remained at the interface, one should have $\Gamma_{\text {elli }}=\Gamma$. Note that $\Gamma_{\text {elli }}<\Gamma$ could also signify loss of particles into bulk water. 
These discrepancies indicate that the approximation made of an interfacial layer of particles surrounded by water cannot be used. A better approximation should involve two layers: the first being the upper part of the particles and air, the second being the lower part of the particles and water (Figure 6). The relative thickness of the two layers is related to the contact angle of the particles with water.

\subsubsection{Ellipsometry - a two-layer model}

Recently, ${ }^{40}$ such a two-layer model was proposed by Hunter et al. to measure the contact angle of polystyrene latex particles at the air-water interface. However, they were not able to perform ellipsometric scans around the pseudo Brewster angle (where $\Psi$ tends to zero and $\Delta$ switches from $180^{\circ}$ to $0^{\circ}$ or $360^{\circ}$ ) and in order to fit their data several assumptions were made, including hexagonal packing of particles at the interface. In order to better approximate the real conditions, we also propose a two-layer model, describing the air-silica and silicawater layers, which takes into account the finite contact angle $\theta$ of spherical particles or aggregates at the air-water interface (see Figure 6). In this two-layer model, we have defined three parameters $R, h$ and $\phi . R$ is the radius of the spherical interfacial aggregates, $h$ is the distance from the centre of the particle to the air-water interface (we adopted the convention $h$ $>0$ for $\theta \leq 90^{\circ}$, and $h<0$ if $\theta>90^{\circ}$ ), and $\phi$ is the surface coverage of particles in the interfacial layer (= total particle cross-sectional area/total area).

In the following, two effective medium approximations (EMA) were used to describe the dielectric constant of the two layers. This type of approximation allows to accurately evaluate the dielectric properties of a heterogeneous layer. ${ }^{41}$ Furthermore, EMA methods were also verified for Langmuir-Blodgett silica nanoparticle layers. ${ }^{42}$ We first assumed a linear change of the dielectric constant with concentration (Wiener effective medium approximation). ${ }^{41}$ 
Since the layers contain spherical inclusions, we also tried the Maxwell-Garnett EMA, expected to be even more accurate. ${ }^{41}$

\subsubsection{Two-layer models. Effective medium approximations}

Wiener effective medium approximation. If the change of dielectric constant with the concentration is linear, the first layer is simply characterized by a thickness $R-h$ and a dielectric constant:

$\varepsilon_{l, 1}=\phi \cdot \frac{V_{s a}}{V_{c a}} \cdot \varepsilon_{S i O 2}+\left(1-\phi \cdot \frac{V_{s a}}{V_{c a}}\right) \cdot \varepsilon_{A i r} ;$

and the second layer by a thickness $R+h$ and a dielectric constant:

$\varepsilon_{l, 2}=\phi \cdot \frac{V_{s w}}{V_{c w}} \cdot \varepsilon_{S i O 2}+\left(1-\phi \cdot \frac{V_{s w}}{V_{c w}}\right) \cdot \varepsilon_{H 2 O}$

where $V_{s a}\left(=\pi\left[2 / 3 R^{3}-\left(R^{2} h-h^{3} / 3\right)\right]\right)$ and $V_{s w}\left(=\pi\left[2 / 3 R^{3}+\left(R^{2} h-h^{3} / 3\right)\right]\right)$ are the portions of volume occupied by a silica sphere in air and water respectively (see Figure 6) and $V_{c a}=\pi R^{2}$ $(R-h), V_{c w}=\pi R^{2}(R+h)$ being the volume of the corresponding cylinders of base $\pi R^{2}(=$ particle cross-section). Thus, in this model and in the following ones, we reduced the unknown parameters to just three: $\phi, h$ and $R$.

Maxwell-Garnett effective medium approximation. For a layer where one can consider a major (host) component (A) and a dispersed spherical component (B) $\varepsilon_{l}$ is given by the MaxwellGarnett relation:

$\varepsilon_{l}=\varepsilon_{A} \frac{\varepsilon_{B}\left(1+2 f_{B}\right)-\varepsilon_{A}\left(2 f_{B}-2\right)}{\varepsilon_{A}\left(2+f_{B}\right)-\varepsilon_{B}\left(1-f_{B}\right)}$ 
where $f_{A}$ and $f_{B}$ are the volume fractions, i.e. $f_{A}+f_{B}=1$. Eq. 5 is valid when the spherical dispersed component is completely surrounded by the host component.

We applied Eq. 5 for each of the two layers described above. The silica medium is component B. For the first layer, component $\mathrm{A}$ is air and $f_{B}=\phi V_{s a} V_{c a}$. For the second layer, component A is water and $f_{B}=\phi V_{s w /} V_{c w}$. Refractive indexes $n_{l}=\sqrt{\varepsilon_{l}}$ can be calculated from Eqs. 3 to 5 . In order to perform the fits of the ellipsometric scans, we implemented an algorithm in Origin (OriginLab Corporation, USA) using the programming language OriginC.

\subsubsection{Two layer model - Deposited layers}

MAI ellipsometry allows evaluating just two parameters and in our problem the unknown ones are $\phi, h$ and R. Several hypotheses can be made in order to fix one of the unknown parameters. The most reasonable hypothesis remains $\Gamma_{\text {elli }}=\Gamma$. If we assume that the added material does not move into the bulk phase, the following relation holds: $\Gamma_{\text {elli }}=4 / 3 \phi \rho R$, where $\rho$ is the bulk density of the particle $\left(2200 \mathrm{~kg} / \mathrm{m}^{3}\right.$ for amorphous silica). Now we can write the surface coverage $\phi$ as a function of $R$. Thus, it is possible to fit just $R$ and $h$ imposing

$$
\phi=3 / 4 \Gamma_{\mathrm{elli}} /(\rho R)
$$

Using the EMA we obtained fits of high quality for the whole set of measurements. The results of the fits are summarized in Table 1. Fits using Wiener and Maxwell-Garnett approximations are in reasonable agreement with each other and the results give a consistent picture of the interfacial structure. Note that no satisfying fit can be obtained assuming the presence of only primary particles of $20 \mathrm{~nm}$ diameter. In fact, if $R=10 \mathrm{~nm}$, around the Brewster angle, $\Delta$ would change from $180^{\circ}$ to $360^{\circ}$ instead of from $180^{\circ}$ to $0^{\circ}$. 
For these layers we observe that by increasing $\Gamma$, the thickness of the layer and the surface coverage increase. For $62 \%$ and $34 \% \mathrm{SiOH}$ layers the immersion thickness $h$ also increases, suggesting that the particles move into water. However, for the $20 \% \mathrm{SiOH}$ layer it seems that the particles move into air. The surface coverage $\phi$ of the interfacial aggregates at $\Gamma=160 \mathrm{mg} \mathrm{m}^{-2}$ is close to the fully packed value $(=0.9)$. Furthermore, increasing $\Gamma$ from 40 to $80 \mathrm{mg} \mathrm{m}^{-2}$, a significant increase of $\phi$ can be related to the surface pressure increase from 5 to $20 \mathrm{mN} \mathrm{m}^{-1}$ displayed in Figure 1 .

For the $34 \% \mathrm{SiOH}$ layer the value of $R$ is really close to the value of the hydrodynamic radius of particles in bulk as measured by dynamic light scattering. For $20 \%$ and $62 \% \mathrm{SiOH}$ layers $R$ is lower than the bulk values being $c a .30-60 \mathrm{~nm}$. It is possible that these particles are less spherical than those for $34 \% \mathrm{SiOH}$ or that the bulk aggregates partially break up at the surface. Negligible changes of the ellipsometric parameters with time were observed for deposited layers. They do not depend either on the location in the surface where the measurements are made (note that they cannot be made close to the trough boundaries where we sometimes see thicker layers, see Figure 4).

\subsubsection{Two layer model - Compressed layers}

Turning now to compressed layers in a Langmuir trough, Figure 7 shows $\Psi$ and $\Delta$ for particle layers of $34 \% \mathrm{SiOH}$ at different nominal concentrations compressed and relaxed $(t \sim$ $\left.10^{4} \mathrm{~s}\right)$. For $\Gamma=20 \mathrm{mg} \mathrm{m}^{-2}, \Psi$ and $\Delta$ do not differ significantly from the bare air-water interface. Increasing the surface concentration, the ellipsometric angles change dramatically. We analyzed the data in the framework of the two-layer model introduced previously using EMA and we summarize our results in Table 2. For the smallest surface concentration measured, $\Gamma=20 \mathrm{mg} \mathrm{m}^{-2}, \Psi$ and $\Delta$ vary rapidly around the Brewster angle and the fitting 
parameters show some inaccuracy. Above $\Gamma=30 \mathrm{mg} \mathrm{m}^{-2}$ and up to $\Gamma=60 \mathrm{mg} \mathrm{m}^{-2}$, the height of the particle layer switched from positive to negative and the surface coverage $\phi$ changes dramatically from $c a$. 0.2 to 0.7 (as was observed by BAM, see Figure 2). This suggests that the particles move into the air. Note that the measurements were performed after the disappearance of buckling $\left(10^{4} \mathrm{~s}\right)$.

For $\Gamma=100 \mathrm{mg} \mathrm{m}^{-2}, \phi=0.83$ and $h=-38 \mathrm{~nm}$ (at $t \sim 10^{4} \mathrm{~s}$, within the Wiener approximation) are evaluated meaning that the particle layer is almost fully packed with the particles placed mostly on the air side. Finally, we compare our data measured at $t \sim 10^{4}$ and $10^{5} \mathrm{~s}$ (see Table 2) to see if the changes in pressure observed in Figure 3 can be similarly observed by ellipsometry. These changes are particularly evident for $\Gamma=100 \mathrm{mg} \mathrm{m}^{-2}$, where the ellipsometric parameters change dramatically and the height and the coverage change from -38 to $-27 \mathrm{~nm}$ and from 0.83 to 0.77 respectively.

The contact angle of the particles with water can be also calculated as: $\theta=$ $\arccos (h / R)$. We know that at high concentration $\Gamma$, the compressed layers buckle, but that after some time, it becomes homogeneous again. We cannot exclude the fact that some of the particles could move away from the imaging area, possibly collecting in the trough boundaries. Similarly, thicker regions were seen in the deposited layers at high concentrations. The data for concentrations higher than $50 \mathrm{mg} \mathrm{m}^{-2}$ therefore need to be analysed with caution. In order to evaluate the contact angle, we have only used the data at lower concentrations. We found that $\theta$ is $57^{\circ}$ with the Maxwell-Garnett approximation $\left(55^{\circ}\right.$ with the Wiener approximation) for $30 \mathrm{mg} \mathrm{m}^{-2}$ and $56^{\circ}$ (resp. $54^{\circ}$ ) for $40 \mathrm{mg} \mathrm{m}^{-2}$. These values are in good agreement with the angles deduced for the deposited layer $51^{\circ}\left(\right.$ resp. $\left.48^{\circ}\right)$. The contact angle increases with increasing particle hydrophobicity up to $114^{\circ}$ for $20 \% \mathrm{SiOH}$ layers (with the Maxwell-Garnett approximation, $112^{\circ}$ with the Wiener approximation). 
These results are in reasonable agreement with the values reported experimentally on macroscopic substrates of pressed powders of the same particles ${ }^{43}$ and the ones calculated theoretically using surface energy considerations. ${ }^{44}$ The value calculated for the $62 \% \mathrm{SiOH}$ is less accurate due to the sharp profile and the absence of measurements very close to the Brewster angle. Except for this particular case, MAI ellipsometry appears as a powerful tool to measure directly the contact angle of solid particles at the air-water interface.

\section{Summary and conclusions}

We have measured the surface pressures of silica nanoparticle layers at the air-water surface prepared by two different protocols: compression of the layer in a Langmuir trough and successive spreading. We observed that the surface pressure of the compressed layers is consistently larger than that of deposited layers. At large $\Gamma$, the compressed layers undergo buckling, this being accompanied by a drastic change in slope of the $\Pi-\Gamma$ isotherm. The deposited layers remain more homogeneous at high concentrations, except close to the trough boundaries where thicker layers are seen.

We also studied the particle layers by MAI ellipsometry introducing a two-layer model able to evaluate the interfacial thickness and the contact angle $\theta . \theta$ increases with increasing particle hydrophobicity and is in good agreement with independent determinations. The characteristics of layers containing particles with $34 \%$ SiOH are similar until $\Gamma^{*}=40 \mathrm{mg} / \mathrm{m}^{2}$, independent of the preparation protocol. The thickness of the layers is close to the bulk aggregate diameter (monolayer) and the fraction of surface covered by particles is $c a$. $20 \%$. Above $\Gamma^{*}$, the area covered by compressed layers increases with compression (up to 90\%) and the particles move to the air as buckling occurs. For deposited layers the surface coverage is lower than in compressed layers ( $c a .70 \%)$ and the particles move into the water subphase. 


\section{Acknowledgement}

We thank Wacker-Chemie (Burghausen) for the kind gift of fumed silica powders, Liliane Léger for lending us the Nanofilm ellipsometer and Eric Raspaud for lending us the light scattering apparatus. D.Y. Zang is grateful to the China Scholarship Council, Laboratoire de Physique des Solides, Université Paris XI and Northwestern Polytechnical University for financial support. 


\section{References}

(1) Xu, H.; Lask, M.; Kirkwood, J.; Fuller, G. Langmuir 2007, 23, 4837.

(2) Aveyard, R.; Clint, J. H.; Nees, D.; Quirke, N. Langmuir 2000, 16, 8820.

(3) Miller, R.; Fainerman, V. B.; Kovalchuk, V. I.; Grigoriev, D. O.; Leser, M. E.; Michel, M. P. Adv. Colloid Interface Sci. 2006, 128-130, 17.

(4) Sethumadhavan, G. N.; Nikolov, A. D.; Wasan, D. T. J. Colloid Interface Sci. 2001, 240, 105.

(5) Pieranski, P. Phys. Rev. Lett. 1980, 45, 569.

(6) Gómez-Guzmán, O.; Ruiz-Garcia, J. J. Colloid Interface Sci. 2005, $291,1$.

(7) Lin, B. J.; Chen, L. J. J.Chem.Phys. 2007, 126, 034706.

(8) Schultz, D. G.; Lin, X. M.; Li, D. X.; Gebhardt, J.; Meron, M.; James-Viccaro, P.; Lin, B. H. J. Phys.Chem. 2006, 110, 24522.

(9) Chen, W.; Tan, S.; Ng, T.; Ford, W.; Tong, P. Phys. Rev. Lett. 2005, 95, 218301.

(10) Horozov, T. S.; Aveyard, R.; Binks, B. P.; Clint, J. H. Langmuir 2003, 19, 2822-2829.

(11) Frydel, D.; Dietrich, S.; Oettel, M. Phys. Rev. Lett. 2007, 99, 118302.

(12) Martínez-Lòpez, F.; Cabrerizo-Vilchez, M. A.; Hidalgo-Álvarez, R. J. Colloid Interface Sci. 2000, 232, 303.

(13) Ghezzi, F.; Earnshaw, J. C. J. Phys. Condens. Matter 1997, 9, L517.

(14) Robinson, D. J.; Earnshaw, J. C. Phys. Rev. A 1992, 46, 2045.

(15) Stankiewicz, J.; Cabrerizo-Vilchez, M. A.; Hidalgo-Álvarez, R. Phys. Rev. E 1993, 47, 2663.

(16) Horozov, T. S.; Aveyard, R.; Binks, B. P.; Clint, J. H. Langmuir 2005, 21, 740574012 .

(17) Horvolgyi, Z.; Mate, M.; Daniel, A.; Szalma, J. Colloids Surf. A 1999, 156, 501-507.

(18) Binks, B. P. Curr. Opin. Colloid Interface Sci. 2002, 7, 21.

(19) Haibel, A.; Rack, A.; Banhart, J. Appl.Phys.Lett. 2006, 89, 154102.

(20) Subramaniam, A. B.; Abkarian, M.; Stone, W. A. Nature Materials 2005, 4, 553.

(21) Horozov, T. S.; Binks, B. P.; Aveyard, R.; Clint, J. H. Colloids Surf. A 2006, 282-283, 377.

(22) Safouane, M.; Langevin, D.; Binks, B. P. Langmuir 2007, 23, 11546.

(23) Stocco, A.; Drenckhan, W.; Rio, E.; Langevin, D.; Binks, B. P. Soft Matter 2009, 5, 2215-2222.

(24) Alargova, R. G.; Warhadpande, D. S.; Paunov, V. N.; Velev, O. D. Langmuir 2004, 20, 10371.

(25) Binks, B. P.; Horozov, T. S. Angew. Chem. Int. Ed. 2005, 44, 3722.

(26) Fujii, S.; Ryan, A. J.; Armes, S. P. J. Am. Chem. Soc. 2006, 128, 7882.

(27) Gonzenbach, U. T.; Studart, A. R.; Tervoort, E.; Gauckler, L. J. Angew. Chem. Int. Ed. 2006, 45, 3526.

(28) Cervantes-Martinez, A.; Rio, E.; Delon, G.; Saint-Jalmes, A.; Langevin, D.; Binks, B. P. Soft Matter 2008, 4, 1531-1535.

(29) Berne, J.; Pecora, R. Dynamic light scattering; Dover Publications: Mineola, 2000.

(30) Azzam, R.; Bazhara, N. Ellipsometry and polarized light; Elsevier: Amsterdam, 1977.

(31) Keddie, J. L. Curr. Opin. Colloid Interface Sci. 2001, 6, 102-110.

(32) Cicuta, P.; Terentjev, E. M. Eur. Phys. J. E 2005, 16, 147.

(33) Petkov, J. T.; Gurkov, T. D. Langmuir 2000, 16, 3703.

(34) Ferenczi, T. A. M.; Cicuta, P. J. Phys.: Condens. Matter 2005, 17, S3445.

(35) Cicuta, P. J.Colloid Interface Sci. 2007, 308, 93.

(36) Zang, D. Y.; Rio, E.; Langevin, D.; Wei, B.; Binks, B. P. submitted to Eur. Phys. J. E.

(37) DeFeijter, J. A.; Benjamins, J.; Veer, F. A. Biopolymers 1978, 17, 1759. 
(38) Khlebtsov, B. N.; Khanadeev, V. A.; Khlebtsov, N. G. Langmuir 2008, 24, 89648970.

(39) Cuypers, P. A.; Corsel, J. W.; Janssen, M. P.; Kop, J. M. M.; Hermens, W. T.; Hemker, H. C. J. Biol. Chem. 1983, 258, 2426.

(40) Hunter, T. N.; Jameson, G. J.; Wanless, E. J.; Dupin, D.; Armes, S. P. Langmuir 2009, 25, 3440-3449.

(41) Aspnes, D. E. Thin Solid Films 1982, 89, 249.

(42) Nagy, N.; Deak, A.; Horvolgyi, Z.; Fried, M.; Agod, A.; Barsony, I. Langmuir 2006, 22, 8416.

(43) Kostakis, T.; Ettelaie, R.; Murray, B. S. Langmuir 2006, 22, 1273-1280.

(44) Binks, B. P.; Clint, J. H. Langmuir 2002, 18, 1270-1273. 
Table 1. Radius $R$ (fitted), calculated surface coverage $\phi$, height $h$ (fitted) for successively deposited particle layers for different $\% \mathrm{SiOH}$ and different nominal concentration $\Gamma$.

\begin{tabular}{|l|l|l|l|l|l|l|l|}
\hline \multicolumn{4}{|c|}{ Wiener approximation } & \multicolumn{3}{l|}{ Maxwell-Garnett approximation } \\
\hline $\begin{array}{l}\mathrm{SiOH} \\
\%\end{array}$ & $\begin{array}{l}\Gamma / \\
\mathrm{mg} \mathrm{m}^{-2}\end{array}$ & $\begin{array}{l}R / \\
\mathrm{nm}\end{array}$ & $\phi$ & $\begin{array}{l}h / \\
\mathrm{nm}\end{array}$ & $\begin{array}{l}R / \\
\mathrm{nm}\end{array}$ & $\phi$ & $\begin{array}{l}h / \\
\mathrm{nm}\end{array}$ \\
\hline 62 & 40 & $20 \pm 1$ & 0.67 & $5 \pm 3$ & $37 \pm 20$ & 0.36 & $21 \pm 19$ \\
\hline 62 & 80 & $39.2 \pm 0.1$ & 0.69 & $31.0 \pm 0.1$ & $49.2 \pm 0.2$ & 0.55 & $38.6 \pm 0.2$ \\
\hline 62 & 160 & $48.5 \pm 0.2$ & 1.12 & $44.4 \pm 0.2$ & $58.6 \pm 0.4$ & 0.93 & $52.8 \pm 0.7$ \\
\hline 34 & 40 & $65 \pm 1$ & 0.21 & $43.5 \pm 0.8$ & $71 \pm 1$ & 0.19 & $44.8 \pm 0.8$ \\
\hline 34 & 80 & $68.6 \pm 0.7$ & 0.39 & $53.2 \pm 0.6$ & $71.7 \pm 0.7$ & 0.37 & $53.8 \pm 0.5$ \\
\hline 34 & 160 & $80.3 \pm 0.2$ & 0.68 & $69.9 \pm 0.1$ & $82.2 \pm 0.2$ & 0.66 & $70.3 \pm 0.1$ \\
\hline 20 & 40 & $32 \pm 0.1$ & 0.42 & $-1.5 \pm 0.1$ & $31.3 \pm 0.1$ & 0.43 & $-3.1 \pm 0.1$ \\
\hline 20 & 80 & $38.50 \pm 0.01$ & 0.71 & $-14.65 \pm 0.04$ & $35.27 \pm 0.03$ & 0.77 & $-13.1 \pm 0.1$ \\
\hline 20 & 160 & $61.02 \pm 0.02$ & 0.89 & $-41.59 \pm 0.04$ & $56.61 \pm 0.01$ & 0.96 & $-37.9 \pm 0.1$ \\
\hline
\end{tabular}

Table 2. Radius $R$ (fitted), calculated surface coverage $\phi$, height $h$ (fitted) for compressed layers of particles possessing $34 \% \mathrm{SiOH}$ at different nominal concentrations $\Gamma$ and different aging $t$.

\begin{tabular}{|l|l|l|l|l|l|l|l|}
\hline \multicolumn{3}{|c|}{} & \multicolumn{3}{|l|}{ Wiener approximation } & \multicolumn{3}{l|}{ Maxwell-Garnett approximation } \\
\hline$t /$ & $\Gamma /$ & $R /$ & $\phi$ & $\begin{array}{l}h / \\
\mathrm{nm}\end{array}$ & $\begin{array}{l}R / \\
\mathrm{nm}\end{array}$ & $\phi$ & $\begin{array}{l}h / \\
\mathrm{nm}\end{array}$ \\
\hline $10^{4}$ & 20 & 77 (fix) & 0.09 & $70.2 \pm 0.3$ & & & \\
\hline $10^{4}$ & 30 & $42 \pm 23$ & 0.24 & $24 \pm 20$ & $42 \pm 23$ & 0.24 & $23 \pm 20$ \\
\hline $10^{4}$ & 40 & $55 \pm 1$ & 0.24 & $32 \pm 1$ & $64 \pm 1$ & 0.21 & $35.8 \pm 0.7$ \\
\hline $10^{4}$ & 60 & $32.5 \pm 0.1$ & 0.63 & $-11.8 \pm 0.1$ & $28.3 \pm 0.1$ & 0.72 & $-11.3 \pm 0.1$ \\
\hline $10^{5}$ & 60 & $31.90 \pm 0.04$ & 0.64 & $-9.56 \pm 0.06$ & $29.1 \pm 0.1$ & 0.70 & $-8.83 \pm 0.05$ \\
\hline $10^{4}$ & 100 & $41.0 \pm 0.3$ & 0.83 & $-37.6 \pm 0.2$ & $35.2 \pm 0.4$ & 0.96 & $-34.6 \pm 0.2$ \\
\hline $10^{5}$ & 100 & $44.20 \pm 0.02$ & 0.77 & $-27.06 \pm 0.08$ & $39.9 \pm 0.3$ & 0.85 & $-24.70 \pm 0.08$ \\
\hline
\end{tabular}




\section{Figure legends}

Figure 1. Surface pressure as a function of nominal surface concentration for silica particle layers $(34 \% \mathrm{SiOH})$ at the air-water interface. For compressed layers in a Langmuir trough, pressures measured by parallel (solid lines) and perpendicular (dashed lines) Wilhelmy plates at the barrier speed of $25 \mathrm{~cm}^{2} \mathrm{~min}^{-1}$ (black lines) and $7 \mathrm{~cm}^{2} \mathrm{~min}^{-1}$ (grey lines). For deposited layers, parallel (open squares) and perpendicular (filled squares) surface pressures measured in a Langmuir trough and in a circular trough (filled circle).

Figure 2. The textural evolution during compression of a silica nanoparticle layer $(34 \% \mathrm{SiOH})$. $\Gamma=12$ (a), 30 (b), 40 (c) and 50 (d) $\mathrm{mg} \mathrm{m}^{-2}$. The scale bars represent $1 \mathrm{~mm}$.

Figure 3. Textural evolution and surface pressure relaxation $\left(\Pi_{||}\right)$at $\Gamma=60 \mathrm{mg} \mathrm{m}^{-2}$ for particles possessing $34 \% \mathrm{SiOH}$. Scale bars $=1 \mathrm{~mm}$.

Figure 4. The textural evolution during deposition of a silica nanoparticle layer ( $34 \% \mathrm{SiOH}) . \Gamma$ = 22 (a), 44 (b), 61 (c) and 88 (d) $\mathrm{mg} \mathrm{m}^{-2}$. The scale bars represent $1 \mathrm{~mm}$.

Figure 5. For successively deposited particle layers: (a) Ellipsometric scans for particles of different $\% \mathrm{SiOH}$ (given) at the nominal surface concentration $\Gamma=160 \mathrm{mg} \mathrm{m}^{-2}$. Solid lines represent the fits. (b) Calculated ellipsometric surface concentration $\Gamma_{\text {elli }}$ from eq. 1 (black symbols) and eq. 2 (grey symbols) versus nominal surface concentration $\Gamma$. The solid line represents $\Gamma_{\text {elli }}=\Gamma$.

Figure 6. $\quad$ Sketch of silica sphere of radius $R$ and refractive index $n_{S i}$ at the air-water interface. $h$ is the vertical distance between the centre of the sphere and the air-water interface. $V_{s a}$ and $V_{s w}$ represent the volume of that part of the sphere in air and water respectively. $\theta$ is the contact angle of the particle at the air-water interface measured into water.

Figure 7. Ellipsometric scans for $34 \% \mathrm{SiOH}$ particle layers obtained by step compression. Influence of nominal surface concentration $\Gamma$ (given) at $t=10^{4} \mathrm{~s}$. Solid lines represent the fits. 
Figures

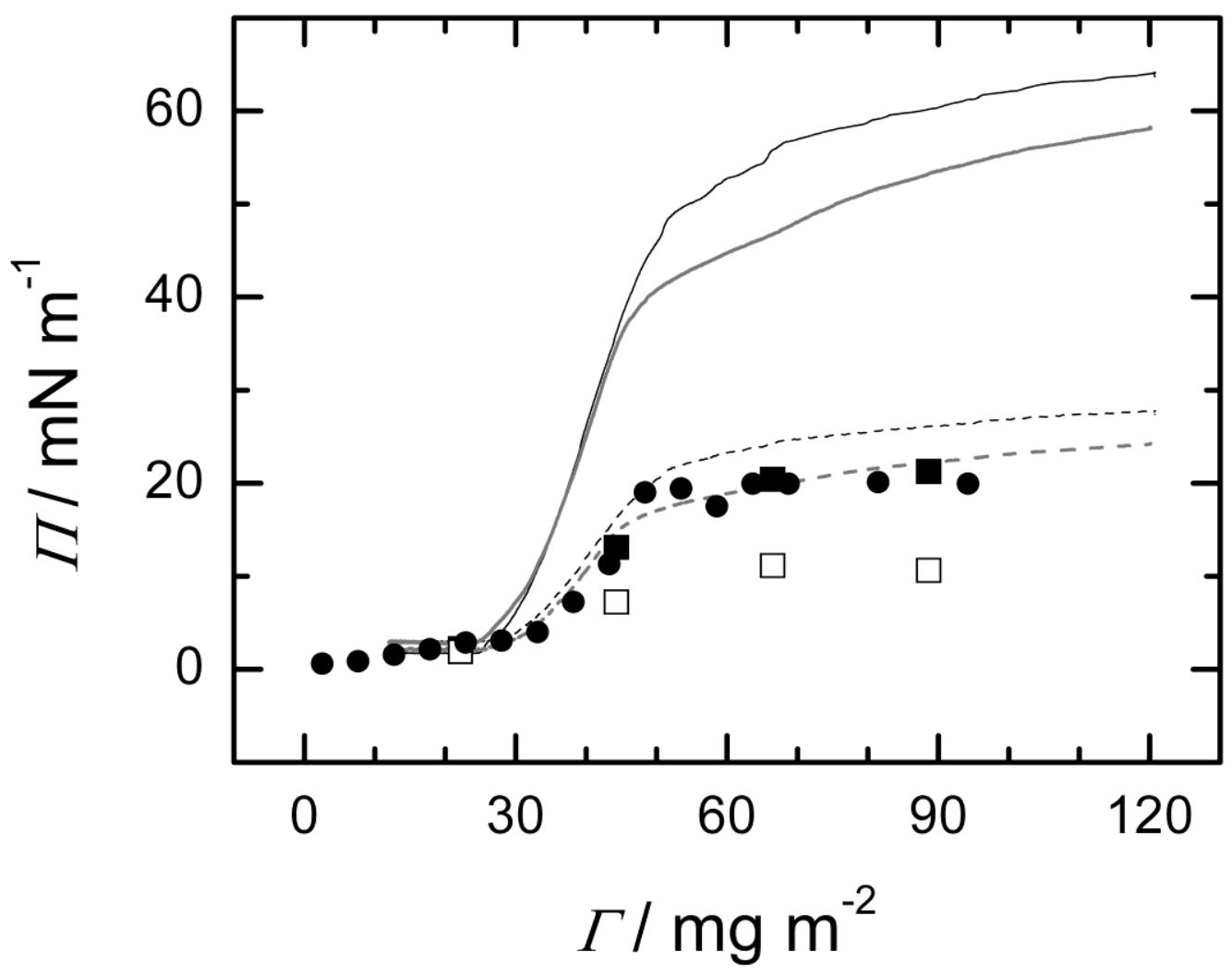

Figure 1 


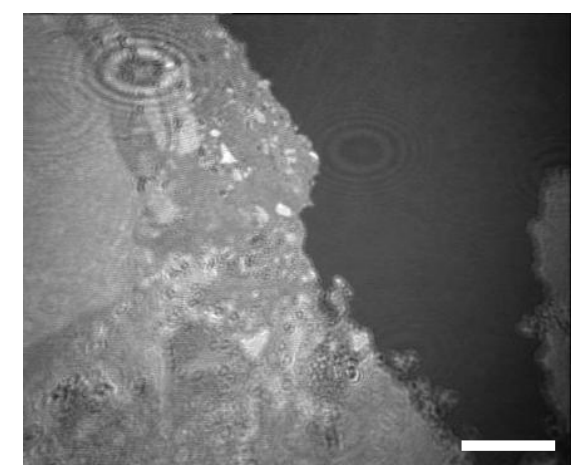

(a)

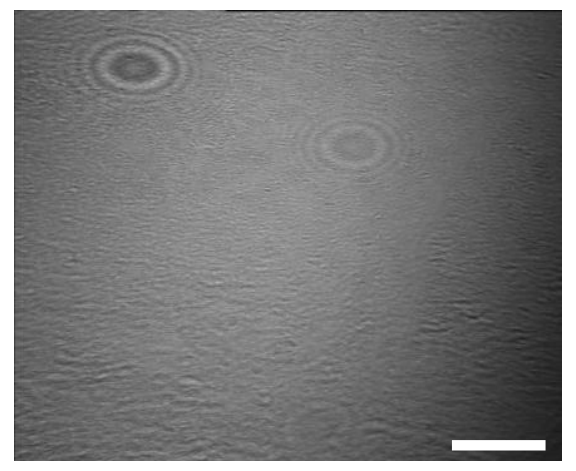

(c)

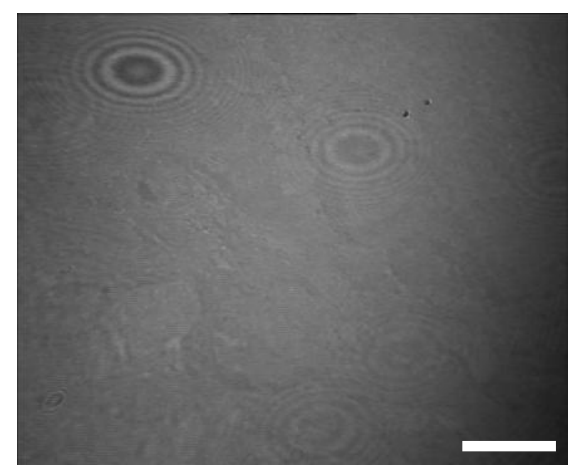

(b)

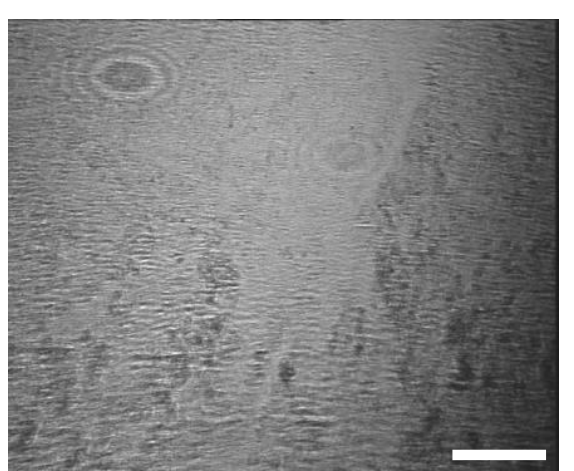

(d)

Figure 2 


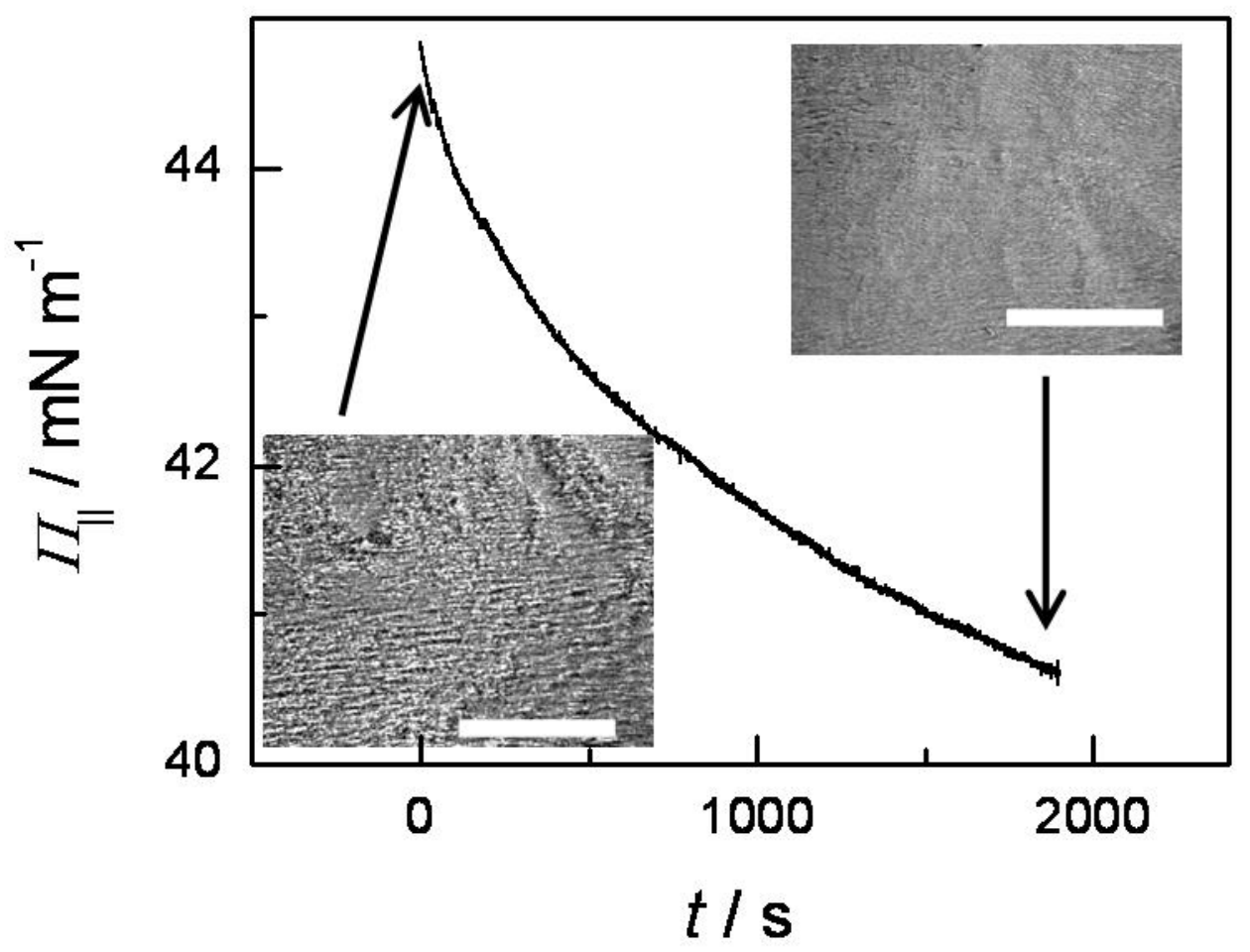

Figure 3 


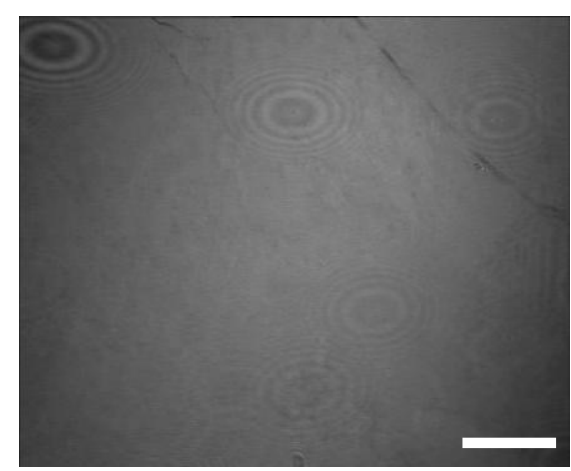

(a)

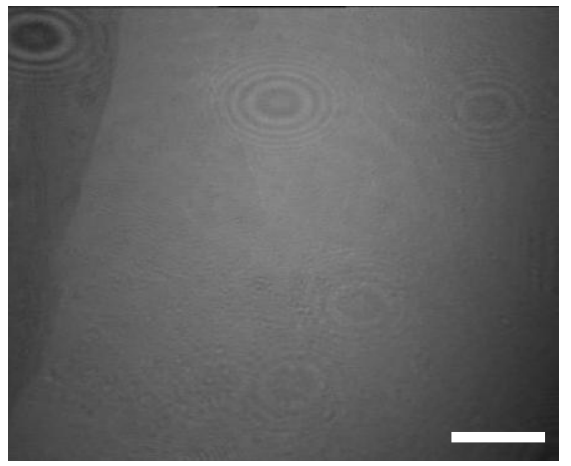

(c)

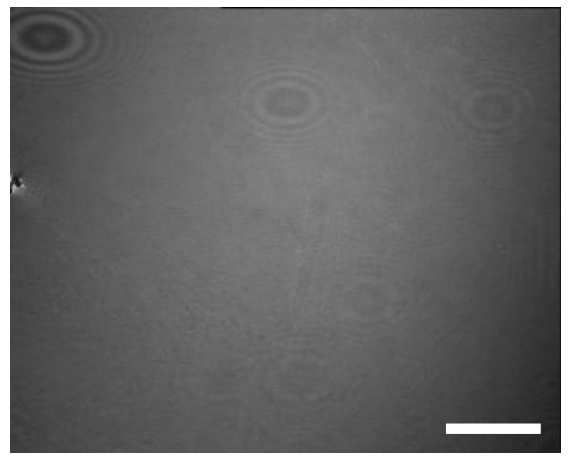

(b)

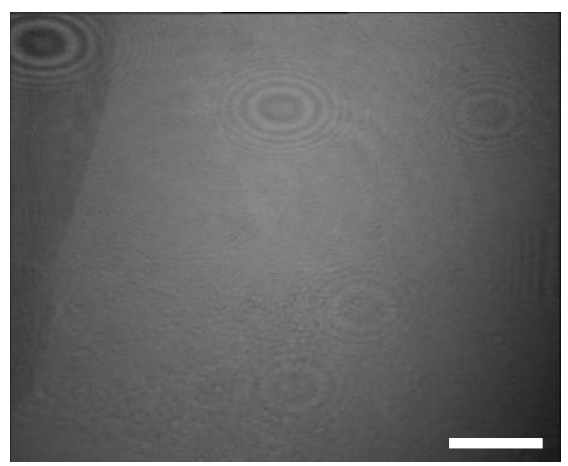

(d)

Figure 4 


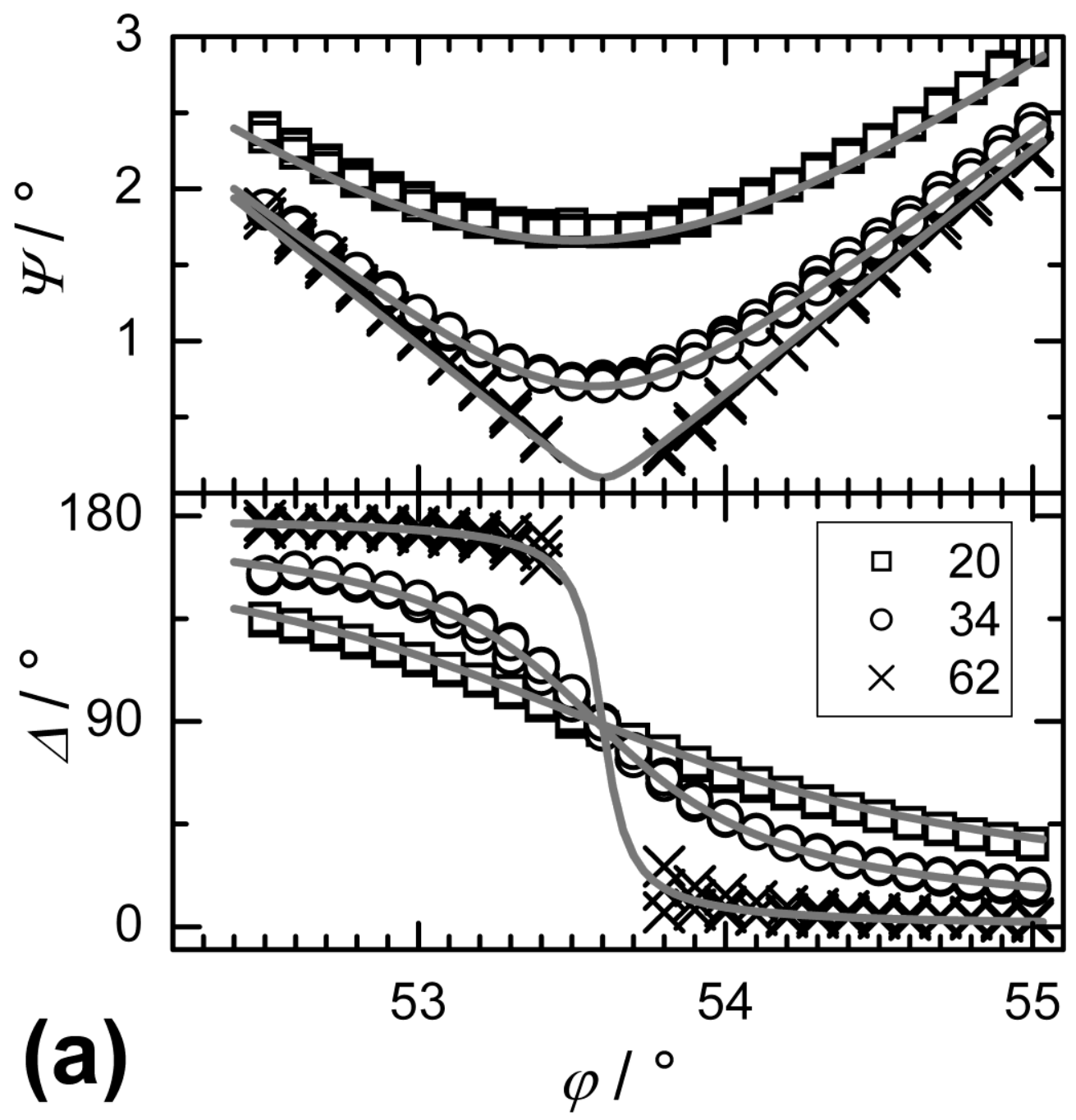

Figure 5a 


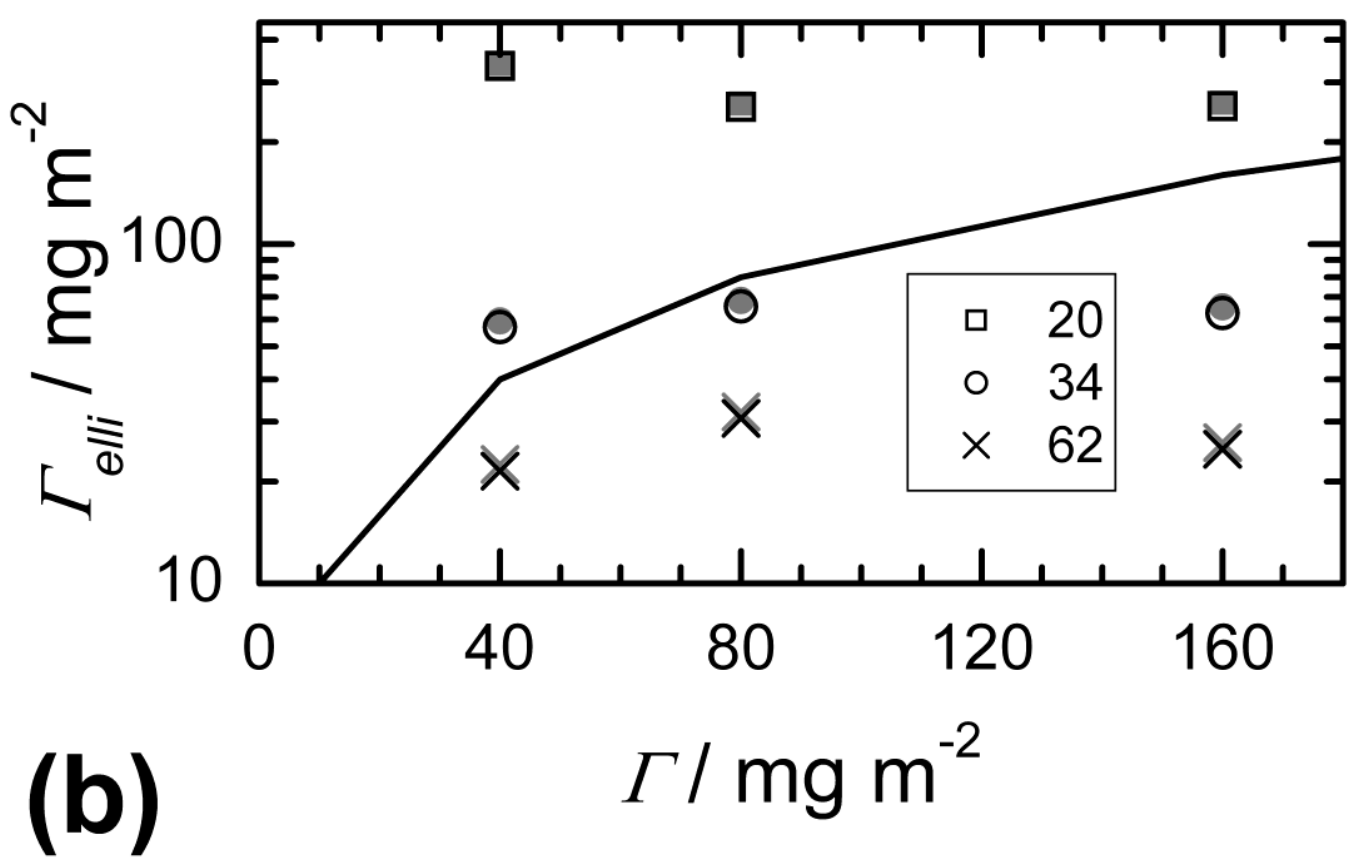

Figure 5b

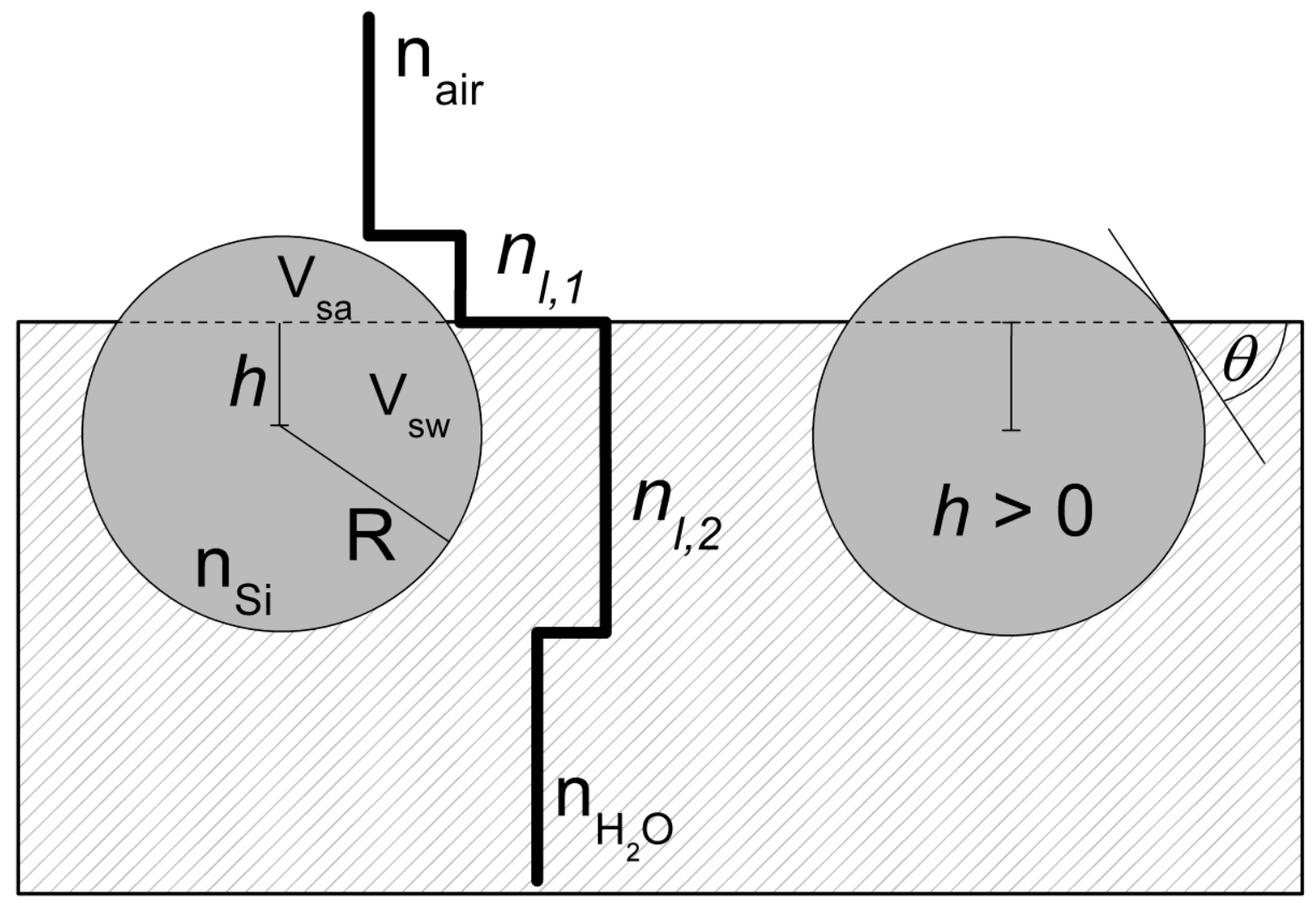

Figure 6 


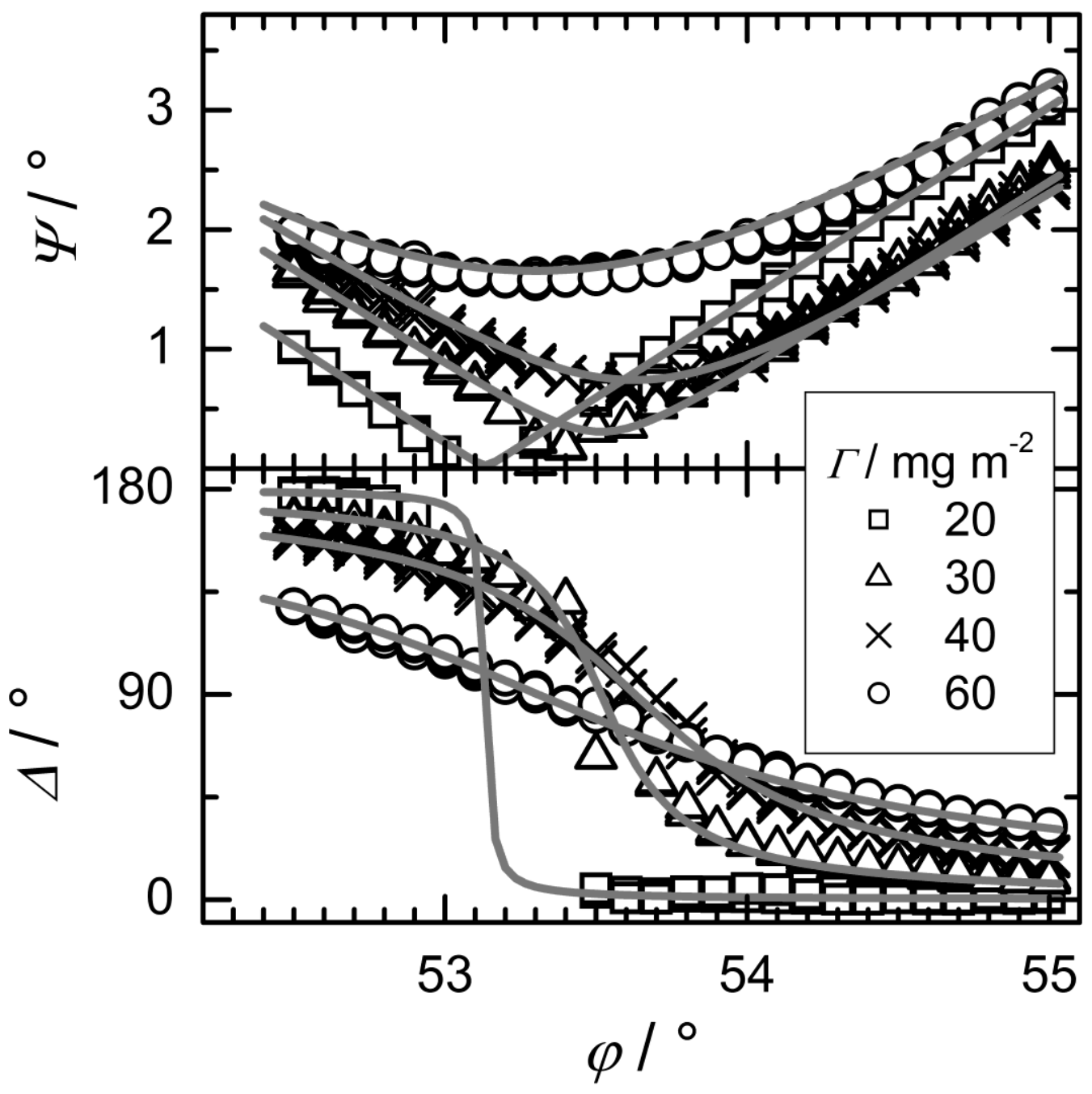

Figure 7 